\title{
Analysis of SVEIR worm attack model with saturated incidence and partial immunization
}

\author{
WANG Fangwei ${ }^{1,2}$, HUANG Wenyan ${ }^{1}$, SHEN Yulong ${ }^{2,3}$, WANG Changguang ${ }^{1}$
}

1. College of Information Technology, Hebei Normal University, Shijiazhuang 050024, China

2. Shannxi Key Laboratory of Network and System Security, Xidian University, Xi'an 710071, China

3. College of Computers, Xidian University, Xi'an 710071, China

\begin{abstract}
Internet worms can propagate across networks at terrifying speeds, reduce network security to a remarkable extent, and cause heavy economic losses. Thus, the rapid elimination of Internet worms using partial immunization becomes a significant matter for sustaining Internet infrastructure. This paper addresses this issue by presenting a novel worm susceptiblevaccinated-exposed-infectious-recovered model, named the SVEIR model. The SVEIR model extends the classical susceptibleexposed-infectious-recovered model (refer to SEIR model) through incorporating a saturated incidence rate and a partial immunization rate. The basic reproduction number in the SVEIR model is obtained. By virtue of the basic reproduction number, we prove the global stabilities of an infection-free equilibrium point and a unique endemic equilibrium point. Numerical methods are used to verify the proposed SVEIR model. Simulation results show that partial immunization is highly effective for eliminating worms, and the SVEIR model is viable for controlling and forecasting Internet worms.
\end{abstract}

Key words: Internet worm, attack model, stability, saturated incidence, partial immunization.

Citation: WANG F W, HUANG W Y, SHEN Y L, et al. Analysis of SVEIR worm attack model with saturated incidence and partial immunization[J]. Journal of communications and information networks, 2016, 1(4): 105-115.

\section{Introduction}

An Internet worm is a type of malicious code capable of duplicating itself and propagating across the Internet. The code red worm in 2001, Slammer worm in 2003, Blaster worm in 2003, Witty worm in 2004, and Conficker worm in 2008 are a few examples of Internet worms, which have caused heavy economic losses and tremendous social panic ${ }^{[1-3]}$. Network experts considered Internet worms the highest security risks on networks ${ }^{[4]}$. Especially, with the development of the IoT (Internet of Things), the threat of Internet worms can be expected to become increasingly serious for network security. Therefore, combating worms is an impending task for defenders. Based on the comparability between malicious worms and biological viruses, numerous mathematical models describing worm propagation have been proposed to study worms' behaviors in the past decade $^{[3,5-8]}$. Mathematical modeling is important in

Manuscript received Aug. 17, 2016; accepted Nov. 28, 2016

This work is supported by the National Natural Science Foundation of China (Nos. 61272541, 61572170), Natural Science Foundation of Hebei Province of China (Nos. F2015205157, F2016205023), Natural Science Foundation of Hebei Normal University (No. L2015Z08), Educational Commission of Hebei Province of China (No. QN2014165). 
determining effective methods against Internet worms in different transmission settings and in quantifying the effects of defending methods. Many models and tools have been proposed to address the dynamic attacking behaviors of worms and effectively counter attacking them under different conditions, e.g., time delay $^{[9]}$, quarantine ${ }^{[10]}$, and antivirus software ${ }^{[11]}$. All the above models are based on the SIR classical epidemic model $^{[12]}$. The SIR model has some drawbacks because it assumes that a susceptible host becomes infectious immediately after contacting with an infected one. Actually, many worms have an exposed period during which susceptible hosts are infected but are not yet contagious. To overcome this drawback, a new model, named the SEIR model, was introduced. The SEIR model incorporates an exposed class ${ }^{[13]}$. Immunization is one of the commonly used methods for controlling and eliminating worms' propagation ${ }^{[14-16]}$. However, all of the models assumed that the vaccine hosts obtained full immunization. This is not consistent with reality. Considering worms' rapid propagation, users or network administrators cannot immunize the entire host population in real networks. In other words, it is very difficult to obtain full immunization for the vaccine hosts. Thus, partial immunization as a fungible and feasible method for eliminating worms has been used for predicting and controlling infectious diseases ${ }^{[17-19]}$. In many worm propagation models, the bilinear infection rate $\beta S I^{[15,16]}$ is used, where, $S$ and $I$ denote the number of susceptible and infectious hosts, respectively. The saturated infection rate $\beta S I /$ $(1+\eta I)$ was firstly introduced by Capasso and Seior ${ }^{[20]}$, where $\beta I /(1+\eta I)$ gravitates towards a saturation value when it becomes large, $1 /(1+\eta I)$ measures the restrain effect of susceptible hosts on the infected hosts $^{[21]}$. The saturated infection rate $\beta S I /(1+\eta I)$ is more rational than the linear rate $\beta S I$. Because it takes the effect of the infected hosts into consideration. The saturated infection rate has subsequently been used in many epidemic models ${ }^{[21-24]}$. This paper proposes a novel SVEIR model based on the SEIR model. Contrary to the existing models, the proposed SVEIR model is armed with partial immunization and saturated infection. Thus, it is a novel worm propagation model with partial immunization. This paper argues that the SVEIR model is appropriate for studying the effects of some security countermeasures on worm propagation. By virtue of the basic reproduction number, we prove the global stabilities of an infection-free equilibrium point and a unique endemic equilibrium. Based on these simulation results, we propose some effective countermeasures for eliminating worms.

This paper is organized as follows: In Section 2, we formulate the extended SVEIR model, which discusses two important factors, i.e., a partial immunization and a saturated incidence rate, and obtains the basic reproduction number. In Section 3 , we resolve the global stability problems of the equilibriums. In Section 4, we provide the simulation results and propose some defending methods. Section 5 concludes the paper.

\section{Mathematical formulation of the SVEIR model}

The SVEIR model extends the classical SEIR model by incorporating a saturated incidence rate and a partial immunization rate. The host population $N$ is divided into five classes and a host at any time $t$ can potentially be in one of the following groups: susceptible, vaccinated, exposed, infectious, recovered, which are denoted by $S, V, E, I, R$, respectively. $S$ is the class of susceptible hosts, $V$ is the class of partially immunizing hosts, $E$ is the class of exposed hosts, $I$ is the class of infective hosts, and $R$ is the class of recovered hosts. The host population $N$ at time $t$ is represented by $N(t)=S(t)+V(t)+E(t)+I(t)$ $+R(t)$. The dynamic transition of the hosts is shown in the following figure. 


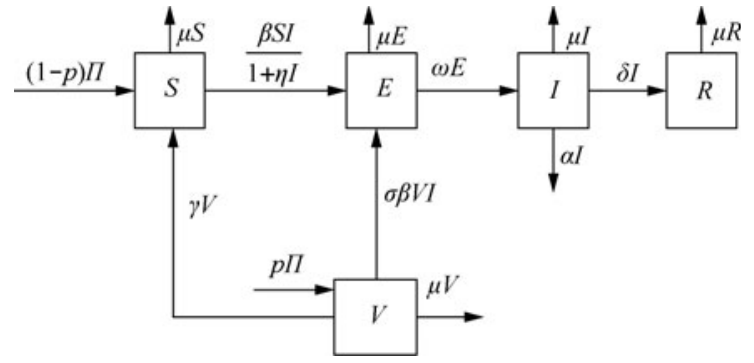

Figure 1 State transition diagram of the SVEIR model

In Fig. $1, \Pi$ is the constant recruitment rate of the host population, $\mu$ denotes the natural death rate of the host population, and $\alpha$ denotes the death rate for a worm attack on infectious hosts. Let $\beta$ be the transmission rate of a worm attack when susceptible hosts have contact with infected ones, $p$ the fraction of recruited hosts that are vaccinated. Let $\gamma$ be the rate at which the vaccine wanes. The emergence of this scenario is due to worm variants. Let $\eta$ represent the parameter measuring the inhibitory effect. Let $\beta S I /(1$ $+\eta I)$ be the saturated infection rate, $\omega$ the rate at which exposed hosts become infectious, and $\sigma$ the recovered rate of infected hosts. Vaccinated hosts which contact infected ones before obtaining immunization, have an infection probability with a transmission rate $\sigma \beta(0 \leqslant \sigma \leqslant 1)^{[18,19]} . \sigma=0$ means that the vaccinated hosts obtain the full immunization, while $\sigma=1$ meaning that the vaccine loses full efficacy to work. Taking some real factors into account, we assume that the vaccinated hosts can obtain partial immunization, i.e. $0<\sigma<1$. According to the previous assumptions, the SVEIR worm propagation model with partial immunization can be described by the following system of differential equations:

$$
\left\{\begin{array}{l}
S^{\prime}(t)=(1-p) \Pi-\frac{\beta S I}{1+\eta I}-\mu S+\gamma V, \\
V^{\prime}(t)=p \Pi-\sigma \beta V I-(\mu+\gamma) V, \\
E^{\prime}(t)=\frac{\beta S I}{1+\eta I}+\sigma \beta V I-(\mu+\omega) E, \\
I^{\prime}(t)=\omega E-(\mu+\alpha+\delta) I, \\
R^{\prime}(t)=\delta I-\mu R .
\end{array}\right.
$$

Since the state $R$ does not appear explicitly in the first four equations in Eq.(1), the dynamics of Eq.(1) is the same as the following system:

$$
\left\{\begin{array}{l}
S^{\prime}(t)=(1-p) \Pi-\frac{\beta S I}{1+\eta I}-\mu S+\gamma V, \\
V^{\prime}(t)=p \Pi-\sigma \beta V I-(\mu+\gamma) V, \\
E^{\prime}(t)=\frac{\beta S I}{1+\eta I}+\sigma \beta V I-(\mu+\omega) E, \\
I^{\prime}(t)=\omega E-(\mu+\alpha+\delta) I .
\end{array}\right.
$$

Our proposed SVEIR model has two aspects that are different from existing models. At the beginning of worm propagation, there is no available vaccine to eliminate infected hosts. The worm propagates with a saturated infection rate $\beta S I /(1+\eta I)$, which is more reasonable than $\beta S I$. Once we detect a worm breakout using an intrusion detection system, we can study the related vaccine capable of defending against such a worm. Some newly arrived hosts are vaccinated through the vaccine, and no longer are infected. As a result, the model reduces the total number of vulnerable hosts and infected hosts.

When summing equations in model in Eq.(2), we have $(S+V+E+I)^{\prime} \leqslant \Pi-\mu(S+V+E+\mathrm{I})$. Then the inequality satisfies that $\lim _{\sup _{\mathrm{t} \rightarrow \infty}}[S(t)+V(t)+E(t)+I(t)] \leqslant \Pi / \mu$, thus the set

$$
\Omega=\left\{(C, V, E, I) \in \mathfrak{R}^{4}: S+V+E+I \leqslant \Pi / \mu\right\}
$$

is positively invariant for model in Eq.(2). As a result, we study the stability condition of the model in Eq.(2) on the set $\Omega$.

It is easily seen that the model in Eq.(2) has an infection-free equilibrium, $P_{0}=\left(S_{0}, V_{0}, 0,0\right)$, where $S_{0}=\frac{\Pi(\mu+\gamma-p \mu)}{\mu(\mu+\gamma)}, V_{0}=\frac{p \Pi}{\mu+\gamma}$. Let $x=(E, I, V, S)^{\mathrm{T}}$, thus the model in Eq.(2) can be represented as

$$
\frac{\mathrm{d} x}{\mathrm{~d} t}=\mathcal{F}(x)-\mathcal{V}(x),
$$

where

$$
\mathcal{F}(x)=\left(\begin{array}{c}
\frac{\beta S I}{1+\eta I}+\sigma \beta V I \\
0 \\
0 \\
0
\end{array}\right),
$$




$$
\mathcal{V}(x)=\left(\begin{array}{c}
(\mu+\omega) E \\
(\mu+\alpha+\delta) I-\omega E \\
\sigma \beta V I+(\mu+\gamma) V-p \Pi \\
\frac{\beta S I}{1+\eta I}+\mu S-(1-p) \Pi-\gamma V
\end{array}\right) .
$$

When differentiating $\mathcal{F}(x)$ and $\mathcal{V}(x)$ with respect to $E, I, V, S$ and computing them at the infectionfree equilibrium $P_{0}=\left(\frac{\Pi(\mu+\gamma-p \mu)}{\mu(\mu+\gamma)}, \frac{p \Pi}{\mu+\gamma}, 0,0\right)$, respectively, we obtain

$$
\begin{gathered}
D \mathcal{F}\left(P_{0}\right)=\left(\begin{array}{cccc}
0 & \beta S_{0}+\sigma \beta V_{0} & 0 & 0 \\
0 & 0 & 0 & 0 \\
0 & 0 & 0 & 0 \\
0 & 0 & 0 & 0
\end{array}\right), \\
D \mathcal{V}\left(P_{0}\right)=\left(\begin{array}{cccc}
\mu+\omega & 0 & 0 & 0 \\
-\omega & \mu+\alpha+\delta & 0 & 0 \\
0 & \sigma \beta V_{0} & \mu+\gamma & 0 \\
0 & -\beta S_{0} & -\gamma & \mu
\end{array}\right) .
\end{gathered}
$$

Therefore, according to Theorem 2 in Ref.[25], the basic reproduction number of the model in Eq.(2), represented by $R_{0}$ is given by

$$
R_{0}=\rho\left(\mathcal{F V}^{-1}\right)=\frac{\omega \beta \Pi\left(\frac{\mu+\gamma-p \mu}{\mu(\mu+\gamma)}+\frac{\sigma p}{\mu+\gamma}\right)}{(\mu+\omega)(\mu+\alpha+\delta)}
$$

\section{Stability analysis for equilibriums in SVEIR}

The endemic equilibrium $P^{*}\left(S^{*}, V^{*}, E^{*}, I^{*}\right)$ of the model in Eq.(2) can be obtained by the following equations:

$$
\left\{\begin{array}{l}
(1-p) \Pi-\frac{\beta S I}{1+\eta I}-\mu S+\gamma V=0, \\
p \Pi-\sigma \beta V I-(\mu+\gamma) V=0, \\
\frac{\beta S I}{1+\eta I}+\sigma \beta V I-(\mu+\omega) E=0, \\
\omega E-(\mu+\alpha+\delta) I=0 .
\end{array}\right.
$$

When solving the model in Eq.(4), we have
$V=\frac{(\mu+\omega)(\mu+\alpha+\delta)}{\omega \sigma \beta}-\frac{B S}{(1+\eta I) \sigma \beta}, E=\frac{(\mu+\alpha+\delta) I}{\omega}$, $I=\frac{p \Pi-(\mu+\gamma) V}{\sigma \beta V}$. Substituting $V$ and $I$ into the first equation of model in Eq.(4) with the foregoing obtained values, we obtain the following equation

$$
(1-p) \Pi-\frac{\beta S \frac{p \Pi-(\mu+\gamma) V}{\sigma \beta V}}{1+\eta I}-\mu S+\gamma A_{1},
$$

where, $A_{1}=\frac{(\mu+\omega)(\mu+\alpha+\delta)}{\omega \sigma \beta}-\frac{B S}{(1+\eta I) \sigma \beta}$.

By a direct algebraic computation, we have

$$
\begin{gathered}
\Pi-\mu S-A_{2}+\mu A_{4} S+A_{3}=0, \\
\text { where } A_{2}=\frac{p \Pi(\mu+\omega)(\mu+\alpha+\delta)}{\frac{\omega \beta S}{1+\eta I}-(\mu+\omega)(\mu+\alpha+\delta)}, \\
A_{3}=\frac{\gamma(\mu+\omega)(\mu+\alpha+\delta)}{\omega \sigma \beta}, A_{4}=\frac{1}{\sigma(1+\eta I)} .
\end{gathered}
$$

Supposing

$$
F(S)=\Pi-\mu S-A_{2}+\mu A_{4} S+\gamma A_{3} .
$$

For $S=0, F(0)=(1-p) \Pi+A_{3}$. It is easily seen that $F(0)>0$.

$$
\begin{aligned}
F^{\prime}(S) & =-\mu-\frac{p \Pi \omega \beta(\mu+\omega)(\mu+\alpha+\delta) \frac{1}{1+\eta I}}{\left(\frac{\omega \beta S}{1+\eta I}-(\mu+\omega)(\mu+\alpha+\delta)\right)^{2}}+\mu A_{4} \\
& <-\mu+\frac{p \Pi \omega \beta \frac{1}{1+\eta I}}{\frac{\omega \beta S}{1+\eta I}-(\mu+\omega)(\mu+\alpha+\delta)}+\mu A_{4} \\
& =-\mu+\left(\frac{\mu}{\sigma}-\frac{p \beta \Pi}{\frac{(\mu+\omega)(\mu+\alpha+\delta)}{\omega}-\frac{\beta S}{1+\eta I}}\right) \frac{1}{1+\eta I}<0 .
\end{aligned}
$$

Therefore, the sign of $F^{\prime}(S)$ is negative. On the other hand, if $R_{0}>1, \omega \beta\left(S_{0}+\sigma V_{0}\right)=$ $\omega \beta\left(S_{0}+\frac{\sigma p \Pi}{\mu+\gamma}\right)>(\mu+\omega)(\mu+\alpha+\delta)$. 


$$
\begin{aligned}
F\left(S_{0}\right) & =\Pi-\mu S_{0}-(\mu+\gamma) A_{3}+\mu A_{4} S_{0}+\gamma A_{3} \\
& <\Pi-\mu S_{0}-\frac{\mu S_{0}}{\sigma(1+\eta I)}-\frac{\mu p \Pi}{\mu+\gamma}+\frac{\mu S_{0}}{\sigma(1+\eta I)} \\
& =\Pi-\mu S_{0}-\frac{\mu p \Pi}{\mu+\gamma}=0 .
\end{aligned}
$$

If $S>S_{0}, F(S)<0$. As a result, the equation $F(S)=0$ only has a root $S^{*}$ which always exists in $\left(0, S_{0}\right)$. When $R_{0} \leqslant 1$, the system in Eq.(2) only has an infection-free equilibrium $P_{0}\left(S_{0}, V_{0}, 0,0\right)$. When $R_{0}>1$, the system in Eq.(2) has the unique endemic equilibrium $P^{*}\left(S^{*}, V^{*}\right.$, $\left.E^{*}, I^{*}\right)$ except for the infection-free equilibrium $P_{0}$.

\subsection{Stability of infection-free equilibrium}

The model in Eq.(2) always has an infection-free equilibrium $P_{0}=\left(\frac{\Pi(\mu+\gamma-p \mu)}{\mu(\mu+\gamma)}, \frac{p \Pi}{\mu+\gamma}, 0,0\right) . P_{0}$ corresponds to the model condition of non-worm breakout. It is important to eliminate worms for defenders.

Proposition 1 For the model in Eq.(2), the infectionfree equilibrium $P_{0}$ is locally asymptotically stable in the set $\Omega$ if $R_{0}<1$.

Proof 1 According to $P_{0}=\left(S_{0}, V_{0}, 0,0\right)$, the Jacobian matrix at $P_{0}$ of the model in Eq.(2) is

$$
J\left(P_{0}\right)=\left(\begin{array}{cccc}
-\mu & \gamma & 0 & -\beta S_{0} \\
0 & -\mu-\gamma & 0 & -\sigma \beta V_{0} \\
0 & 0 & -\mu-\omega & \beta S_{0}+\sigma \beta V_{0} \\
0 & 0 & \omega & -\mu-\alpha-\delta
\end{array}\right) .
$$

We easily obtain that it always has two negative eigenvalues $\lambda_{1}=-\mu$, and $\lambda_{2}=-\mu-\gamma$. The other eigenvalues are decided by the following equation

$$
(\lambda+\mu+\omega)(\lambda+\mu+\alpha+\delta)-\omega\left(\beta S_{0}+\sigma \beta V_{0}\right)=0 .
$$

A simple computation is used to show that Eq. (5) is equal to

$$
\lambda^{2}+(2 \mu+\omega+\alpha+\delta) \lambda+C=0,
$$

where, $C=(\mu+\omega)(\mu+\alpha+\delta)-\omega\left(\beta S_{0}+\sigma \beta V_{0}\right)$.

If $R_{0}<1,(\mu+\omega)(\mu+\alpha+\delta)-\omega\left(\beta S_{0}+\sigma \beta V_{0}\right)>0$, thus the two roots of Eq.(6) are negative. The locally asymptotically stable condition of $P_{0}$ is that $\lambda_{i}<0$, for $i=1,2,3,4$, which meets the sufficient condition of the stability theory ${ }^{[26]}$. When $R_{0}>1,(\mu+\omega)(\mu+\alpha+\delta)$ $-\omega\left(\beta S_{0}+\sigma \beta V_{0}\right) .<0$, which means that $J\left(P_{0}\right)$ has both a positive root and a negative root. Therefore, the infection-free equilibrium $P_{0}$ is an unstable saddle point. This completes the proof.

Proposition 2 For the model in Eq.(2), the infectionfree equilibrium point $P_{0}$ is globally asymptotically stable if $R_{0} \leqslant 1$.

Proof 2 To prove $P_{0}$ is globally asymptotically stable, we construct the following Lyapunov function: $L(E, I)=\omega E+(\mu+\omega) I$.

The derivation of $L(E, I)$ with respect to $t$ gives

$$
\begin{aligned}
L^{\prime}(t) & =\omega E^{\prime}+(\mu+\omega) I^{\prime} \\
& =\frac{\omega \beta S I}{1+\eta I}+\omega \sigma \beta V I-(\mu+\omega)(\mu+\alpha+\delta) I \\
& \leqslant(\omega \beta S+\omega \sigma \beta V-(\mu+\omega)(\mu+\alpha+\delta)) I \\
& =\frac{\omega \beta\left(S_{0}+\sigma V_{0}\right)}{R_{0}}\left(\frac{R_{0}(S+\sigma V)}{S_{0}+\sigma V}-1\right) I \\
& \leqslant 0 .
\end{aligned}
$$

Furthermore, $L^{\prime}=0$ if and only if $I=0$. Thus, the largest compact invariant set in $\left\{(S, V, E, I) \mid L^{\prime}=0\right\}$ is the singleton $\left\{P_{0}\right\}$. When $R_{0} \leqslant 1$, the global stability of $P_{0}$ satisfies LaSalle's invariance principle ${ }^{[27]}$. LaSalle's invariance principle ${ }^{[27]}$ implies that $P_{0}$ is globally asymptotically stable in the set $\Omega$. This completes the proof.

\subsection{Endemic equilibrium and its stability}

From the aforementioned computation, we know that the model in Eq.(2) has the unique endemic equilibrium $P^{*}$. The endemic equilibrium $P^{*}$ means that the worm does not die out when it appears. Finally, every class of the model reaches its stable state. $S^{*}, V^{*}, E^{*}, I^{*}$ and $R^{*}$ are not equal to zero. Next, we investigate the local stability of the endemic 
equilibrium $P^{*}=\left(S^{*}, V^{*}, E^{*}, I^{*}\right)$.

Proposition 3 When $R_{0}>1$, the endemic equilibrium $P^{*}$ is locally asymptotically stable in the region $\Omega$.

Proof 3 The Jacobian matrix of Eq.(2) at the endemic equilibrium $P^{*}$ is

$J\left(P^{*}\right)=\left(\begin{array}{cccc}-D_{1} & \gamma & 0 & -\frac{\beta S^{*}}{\left(1+\eta I^{*}\right)^{2}} \\ 0 & -D_{2} & 0 & -\sigma \beta V^{*} \\ \frac{\beta I^{*}}{1+\eta I^{*}} & \sigma \beta I^{*} & -(\mu+\omega) & D_{3} \\ 0 & 0 & \omega & -(\mu+\alpha+\delta)\end{array}\right)$,

where, $D_{1}=\frac{\beta I^{*}}{1+\eta I^{*}}+\mu, D_{2}=\sigma \beta I^{*}+(\mu+\gamma)$,

$D_{3}=\frac{\beta S^{*}}{\left(1+\eta I^{*}\right)^{2}}+\sigma \beta V^{*}$.

Thus, the corresponding characteristic equation can be described as

$$
\lambda^{4}+C_{1} \lambda^{3}+C_{2} \lambda^{2}+C_{3} \lambda+C_{4}=0
$$

where,

$$
\begin{aligned}
C_{1} & =4 \mu+\alpha+\omega+\delta+\gamma+\sigma \beta I^{*}+\frac{\beta S^{*}}{1+\eta I^{*}}>0, \\
C_{2} & =(\mu+\omega)(\mu+\alpha+\delta)+B_{2}(2 \mu+\omega+\alpha+\delta) \\
& +B_{1}\left(\sigma \beta I^{*}+3 \mu+\gamma+\omega+\alpha+\delta\right)>0, \\
C_{3} & =B_{2}(\mu+\omega)(\mu+\alpha+\delta)+\beta \omega \mu \frac{S^{*}}{\left(1+\eta I^{*}\right)^{2}} \\
& +B_{1}\left[(\mu+\omega)(\mu+\alpha+\delta)+B_{2}(2 \mu+\omega+\alpha+\delta)\right] \\
& \geqslant(\mu+\omega)(\mu+\alpha+\delta)\left(\sigma \beta I^{*}+2 \mu+\gamma+\frac{\beta I^{*}}{1+\eta I^{*}}\right) \\
& +B_{1} B_{2}(2 \mu+\omega+\alpha+\delta)>0, \\
C_{4} & =B_{1} B_{2}(\mu+\alpha+\delta)+\gamma \omega \mu \sigma \beta V^{*}+\beta \omega \mu \frac{S^{*}}{\left(1+\eta I^{*}\right)^{2}} B_{2} \\
& \geqslant B_{1} B_{2}(\mu+\omega)(\mu+\alpha+\delta)+\gamma \omega \mu \sigma \beta V^{*}>0 .
\end{aligned}
$$

Through a simple computation, we obtain that $H_{1}=C_{1}>0, H_{2}=C_{1} C_{2}-C_{3}>0, H_{3}=C_{3} H_{2}-C_{1}{ }^{2} C_{4}>0$, $\mathrm{H}_{4}=\mathrm{C}_{3} \mathrm{H}_{3}>0$.

According to the theorem of Routh-Hurwitz ${ }^{[28]}$, if follows that the endemic equilibrium $P^{*}$ is locally asymptotically stable. From the above discussion, we summarize the following conclusion.

In what follows, we use the geometrical approach ${ }^{[29]}$ to study the stability of the endemic endemic equilibrium $P^{*}$.

Theorem 1 Consider the following systems ${ }^{[27]}$ :

$$
x^{\prime}=f(x), x \in \Omega,
$$

and its corresponding periodic linear system

$$
z^{\prime}=\frac{\partial f^{[2]}}{\partial x} p(t) z(t)
$$

where, $\partial f^{[2]} / \partial x$ is the second additive compound matrix of $\partial f / \partial x$ and $\Theta=\{p(t): 0 \leqslant t \leqslant \omega\}$ is the period orbit of Eq.(8).

We make the following four assumptions:

1) there is a compact absorbing set $K \subset \Omega$ and a unique equilibrium $\bar{x} \in D$;

2) model in Eq.(8) satisfies the Poincare-Bendixson property;

3) Eq.(9) is asymptotically stable for each periodic solution $x=p(t)$ to Eq.(8) with $p(0) \in D$;

$$
\text { 4) }(-1)^{n} \operatorname{det} \frac{\partial f}{\partial x}(\bar{x})>0 \text {, }
$$

Then, the unique equilibrium $\bar{x}$ of model in Eq.(8) is globally asymptotically stable in the set $\Omega$.

Proposition 4 If $R_{0}>1$, the endemic positive equilibrium $P^{*}$ is globally asymptotically stable in the set $\Omega$.

Proof 4 If $R_{0}>1$, model in Eq.(2) is uniformly permanent, and the unique positive equilibrium $P^{*}$ is locally asymptotically stable according to Proposition 3 . Then the infection-free equilibrium point is unstable according to Proposition 1. Furthermore, there exists a compact absorbing set $K \subset \Omega$. Therefore, assumption Eq.(1) holds.

The Jacobian matrix of the model in Eq.(2) is denoted by

$$
J(P)=\left(\begin{array}{cccc}
-G_{1}-\mu & \gamma & 0 & -G_{2} \\
0 & -D_{4} & 0 & -G_{3} \\
G_{1} & \sigma \beta I & 0-(\mu+\omega) & G_{2}+G_{3} \\
0 & 0 & \omega & -G_{5}
\end{array}\right),
$$


where, $G_{1}=\beta I /(1+\eta I), G_{2}=\beta I /(1+\eta I)^{2}, G_{3}=\sigma \beta V$, $G_{4}=\sigma \beta I+(\mu+\gamma), G_{5}=\mu+\alpha+\delta$.

We choose the matrix $H$ with $H=\operatorname{diag}(-1,-1,1$, $-1)$. It is easily proven that all off-diagonal elements of $H J(P) H$ are not positive. Thus, the model in Eq.(2) is competitive. Therefore, assumption 2) holds.

Through an elementary row transformation of the matrix $J(P)$, we obtain

$$
J(P)=\left(\begin{array}{cccc}
-G_{1}-\mu & \gamma & 0 & -G_{2} \\
0 & -D_{4} & 0 & -G_{3} \\
-\mu & -\mu & -(\mu+\omega) & 0 \\
0 & 0 & \omega & -G_{5}
\end{array}\right) .
$$

According to Proposition in Ref.[30], the second additive compound matrix $J^{[2]}(P)$ of $J(P)$ can be denoted by

$$
J(P)=\left(\begin{array}{cccccc}
-E_{1} & 0 & -\sigma \beta V & 0 & G_{2} & 0 \\
-\mu & E_{2} & 0 & \gamma & 0 & G_{2} \\
0 & \omega & E_{3} & 0 & \gamma & 0 \\
\mu & 0 & 0 & E_{4} & 0 & \sigma \beta V \\
0 & 0 & 0 & \omega & E_{5} & 0 \\
0 & 0 & -\mu & 0 & -\mu & E_{6}
\end{array}\right),
$$

where,

$$
\begin{aligned}
& E_{1}=-\left(\frac{\beta I}{1+\eta I}+2 \mu+\sigma \beta I+\gamma\right), E_{2}=-\left(\frac{\beta I}{1+\eta I}+2 \mu+\omega\right), \\
& E_{3}=-\left(\frac{\beta I}{1+\eta I}+2 \mu+\alpha+\sigma\right), E_{4}=-(\sigma \beta I+2 \mu+\gamma+\omega), \\
& E_{5}=-(\sigma \beta I+2 \mu+\gamma+\alpha+\delta), E_{6}=-(2 \mu+\omega+\alpha+\delta) .
\end{aligned}
$$

The second compound system of the model in Eq.(2) within a periodic solution is described by

$$
\left\{\begin{array}{l}
X^{\prime}(t)=-E_{1} X+\sigma \beta V Z+G_{2} M, \\
Y^{\prime}(t)=-\mu X-E_{2} Y+\gamma L-\beta_{1} G_{2} U, \\
Z^{\prime}(t)=\omega Y-\left(\frac{\beta I}{1+\eta I}+2 \mu+\alpha+\delta\right) Z+\gamma M, \\
L^{\prime}(t)=\mu X+(\sigma \beta I+2 \mu+\gamma+\omega) L+\sigma \beta V U, \\
M^{\prime}(t)=\omega L+(\sigma \beta I+2 \mu+\gamma+\alpha+\delta) M, \\
U^{\prime}(t)=-\mu Z-\mu M-(2 \mu+\omega+\alpha+\delta) U .
\end{array}\right.
$$

To verify that the system in Eq.(10) is asymptotically stable, we choose the following Lyapunov function:

$$
\begin{aligned}
& V(X, Y, Z, L, M, U ; S, V, E, I) \\
& =\sup \left\{|X|+|Y|+|L|, \frac{E}{I}(|Z|+|M|+|U|)\right\} .
\end{aligned}
$$

Using the uniform persistence, we know that there is a positive distance between the orbit of $P(t)=(S(t)$, $V(t), E(t), I(t))$ and the boundary of $\Omega$. As a result, there exists a constant $c$ satisfying the following inequality:

$$
\begin{aligned}
& V(X, Y, Z, L, M, U ; S, V, E, I) \\
& \geqslant c \sup \{|X|,|Y|,|Z|,|L|,|M|,|U|\},
\end{aligned}
$$

for all $(X, Y, Z, L, M, U) \in \mathbb{R}^{6}$ and $(S, V, E, I) \in P(t)$.

Let $\phi=\{\gamma, \omega\}$. By direct computations, we obtain the following inequalities:

$$
\begin{aligned}
& D_{+}|X(t)| \leqslant-E_{1} X+\frac{\beta S}{(1+\eta I)^{2}} M \leqslant-(2 \mu+\phi) \\
&+ \frac{\beta S}{(1+\eta I)^{2}}(|M(t)|+|Z(t)|+U(t) \mid), \\
& D_{+}|Y(t)| \leqslant-(2 \mu+\phi)|Y(t)|+\gamma|L(t)| \\
&+ \frac{\beta S}{(1+\eta I)^{2}}(|M(t)|+|Z(t)|+U(t) \mid), \\
& D_{+}|Z(t)| \leqslant \omega|Y(t)|-(2 \mu+\alpha+\delta)|Z(t)|+\gamma|M(t)|, \\
& D_{+}|L(t)| \leqslant \mu|X(t)|-(2 \mu+\phi)|L(t)|+\sigma \beta V|U(t)|, \\
& D_{+}|M(t)| \leqslant \omega|L(t)|-(2 \mu+\alpha+\delta)|M(t)|-\gamma|M(t)|, \\
& D_{+}|U(t)| \leqslant-(2 \mu+\alpha+\delta)|U(t)| .
\end{aligned}
$$

Therefore, we obtain

$$
\begin{aligned}
& D_{+}(|X|+|Y|+|L|) \leqslant-(2 \mu+\phi)(|X|+|Y|+|L|) \\
& \quad+\frac{E}{I}\left(\frac{\beta S I}{E(1+\eta I)^{2}}+\sigma \beta V \frac{I}{E}\right)(|Z|+|M|+|U|), \\
& D_{+}(|Z|+|M|+|U|) \leqslant \omega(|X|+|Y|+|L|) \\
& \quad-(2 \mu+\alpha+\delta)(|Z|+|M|+|U|) .
\end{aligned}
$$

Then,

$$
\begin{gathered}
D_{+}+\frac{E}{I}(|Z|+|M|+|U|) \leqslant \omega \frac{E}{I}(|X|+|Y|+|L|) \\
+\left(\frac{E^{\prime}}{E}-\frac{I^{\prime}}{I}-2 \mu-\alpha-\delta\right) \frac{E}{I}(|L|+|M|+|U|) .
\end{gathered}
$$

From the pervious formula, we have

$$
D_{+}|V(t)| \leqslant \max \left\{g_{1}(t), g_{2}(t)\right\} V(t),
$$


where,

$$
\begin{aligned}
& g_{1}(t)=-2 \mu-\phi+\beta S \frac{I}{E(1+\eta I)^{2}}+\sigma \beta V \frac{I}{E}, \\
& g_{2}(t)=\omega \frac{E}{I}+\frac{E^{\prime}}{E}-\frac{I^{\prime}}{I}-(2 \mu+\alpha+\delta) .
\end{aligned}
$$

From the model (2), we can obtain

$$
\begin{aligned}
& \frac{E^{\prime}}{E}=\frac{\beta S I}{(1+\eta I)^{2}}+\sigma \beta V \frac{I}{E}-(\mu+\omega), \\
& \frac{I^{\prime}}{I}=\omega \frac{E}{I}-(\mu+\alpha+\delta) .
\end{aligned}
$$

Therefore,

$$
g_{1}(t)=\frac{E^{\prime}}{E}-\mu-(\mu-\phi), g_{2}(t)=\frac{E^{\prime}}{E}-\mu .
$$

If $\gamma \geqslant \omega$, then $\omega^{-} \phi=\omega^{-} \omega=0$. If $\gamma<\omega$, then $\omega^{-} \phi$ $=\omega-\gamma>0$. Under the two cases, we always have $\int_{0}^{\zeta} \sup \left\{g_{1}(t), g_{2}(t)\right\} d t \leqslant\left.\ln E(t)\right|_{0} ^{\varsigma}-\mu \varsigma=-\mu \varsigma<0$ which implies that $(X(t), Y(t), Z(t), L(t), M(t), U(t)) \rightarrow 0$, as $t \rightarrow \infty$. Therefore, the second compound system in Eq.(2) is asymptotically stable. This verifies the assumption 3 ).

Let $J\left(P^{*}\right)$ be the Jacobian matrix of the model in Eq.(2) at $P^{*}$, we obtain

$$
\begin{aligned}
J\left(P^{*}\right) & =\left|\begin{array}{cccc}
-D_{1} & \gamma & 0 & -\frac{\beta S^{*}}{\left(1+\eta I^{*}\right)^{2}} \\
0 & -D_{2} & 0 & \sigma \beta V^{*} \\
-\mu & -\mu & -D_{5} & 0 \\
0 & 0 & \omega & -D_{4}
\end{array}\right| \\
& =\omega\left|\begin{array}{ccc|}
-D_{1} & \gamma & -\frac{\beta S^{*}}{\left(1+\eta I^{*}\right)^{2}} \\
0 & -D_{2} & \sigma \beta V^{*} \\
\mu & \mu & 0
\end{array}\right| \\
& +(\mu+\alpha+\delta)(\mu+\omega)\left|\begin{array}{cc}
-D_{1} & \gamma \\
0 & -D_{2}
\end{array}\right| \\
& =\mu \omega\left[\sigma \beta V^{*}\left(D_{1}+\gamma\right)+\frac{\beta S^{*}}{\left(1+\eta I^{*}\right)^{2}} D_{2}\right] \\
& +(\mu+\alpha+\delta)(\mu+\omega) D_{1} D_{2}>0 .
\end{aligned}
$$

Therefore, $(-1)^{6} \operatorname{det}\left(J\left(P^{*}\right)\right)>0$. This verifies the assumption 4).
We verify all the assumptions of Theorem 1 . Therefore, $P^{*}$ is globally asymptotically stable in $\Omega$.

\subsection{Worm epidemic control}

Proposition 2 indicates that the combination efforts (represented by the formulation of $R_{0}$ ) can eliminate worm prevalence in the real networks. Under the SVEIR propagation model, we investigate how to control the infection-free equilibrium in network administration.

Corollary 1 In order to eliminate worms, the partial immunization rate $\sigma$ should satisfy

$$
\sigma \leqslant \frac{(\mu+\omega)(\mu+\alpha+\delta)(\mu+\gamma)}{p \omega \beta \Pi}-\frac{\mu+\gamma-p \mu}{p \mu} .
$$

Proof 5 Using both Eq.(3) and Proposition 2, this corollary holds.

\section{Numerical simulations}

This section develops numerical experimental steps to examine our model and evaluates the effect of the implemented methods. It is difficult to adopt realistic parameters or real network traffic for our study, because many parameters in previous models are assumed according to their hypothesis. We choose the total host population $N=1000000$. The average scan rate of the Slammer worm is $s=4000$ per second $^{[2]}$. The infection rate of the Slammer worm is $\beta=s / 2^{32}=9.3 \times 10^{-7}$. We take proper values of $\Pi$ and $\mu$ so that $\Pi / \mu=N$, implying that the total number of hosts remains unchanged. Therefore, we set $\Pi=100$ and $\mu=0.0001 . \delta=0.4, \omega=0.02, \gamma=0.01, S(0)=999$, 985, $V(0)=10, E(0)=0, I(0)=5, R(0)=0, \eta=2, p=$ $0.2, \alpha=0.0001, \sigma=0.05$. Using the above parameters, we can obtain the basic reproduction number $R_{0}=$ $0.908<1$. The worm should gradually be eliminated according to Proposition 1 and 2. Fig.2 illustrates the change trend of susceptible, infected and recovered 
hosts when $R_{0}$ is 0.908 , respectively. From Fig.2, it is clearly seen that the worm propagation is depressive, which is consistent with an analysis of the theory. Finally, all the infected hosts vanish, and reach a recovered state.

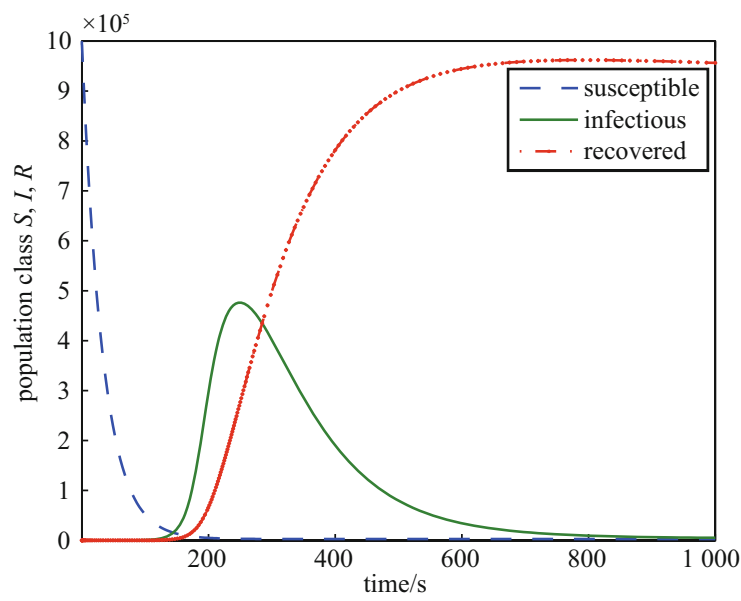

Figure 2 Globally stable infection-free equilibrium

In the second experiment, we change some related parameters about to guarantee $R_{0}>1$. When $p=0.4$ and $\delta=0.003$, we have $R_{0}=9.847>1$. The other parameters remain unchanged. The simulation results are shown in Fig.3, where it can be seen that the number of susceptible, infected and recovered hosts eventually maintain positive values between 0 and $\Pi / \mu$, which indicates that the worm does not

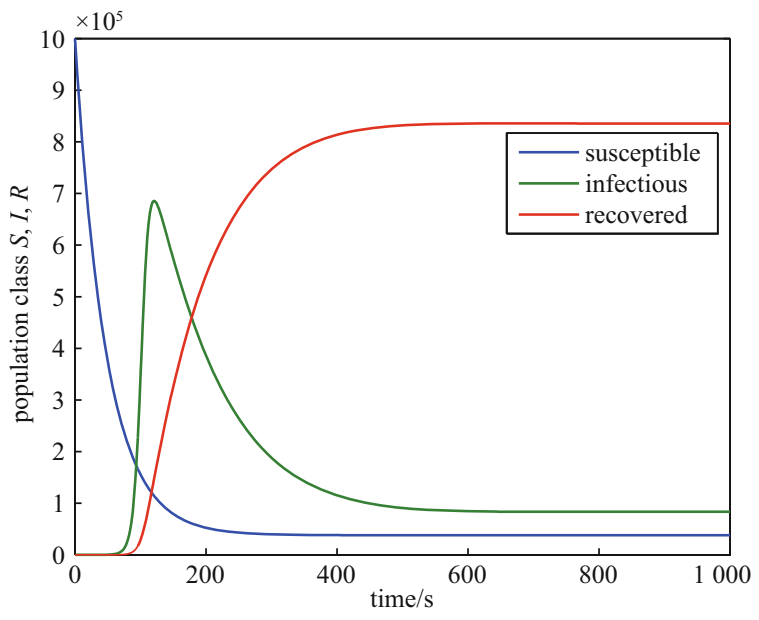

Figure 3 Globally stable endemic equilibrium vanish, if worms are initially present. Finally, these three states reach their equilibrium points $P^{*}=(38094$, 83 543, 83 528), which is consistent with Proposition 3 and 4 . The unique endemic equilibrium $P^{*}$ is globally asymptotically stable if the basic reproduction number is larger than unity.

To demonstrate that the effect of the partial immunization rate on infected hosts, we set the partial immunization rate $\sigma$ to different values. The others parameters remain the same. Fig.4 shows that the effect of changing the partial immunization rate on worm propagations when $\sigma=0,0.1,0.3$, $0.5,0.7$, respectively. Fig.4 shows that no hosts are infected when $\sigma=0$, which means that all hosts gain full immunization. However, in realworld networks, it is very difficult to implement full immunization. As expected, a smaller partial immunization rate results in slowing down the worm propagation speed, more importantly, and decreasing the total number of infected hosts. The partial immunization rate $\sigma$ is related to many factors, such as the performance of network security devices, professional knowledge of the network administrator, and the security consciousness of users. As a result, in order to remove worms as soon as possible, we require the support from all circles of society. Once the vaccine has been studied, computer users should

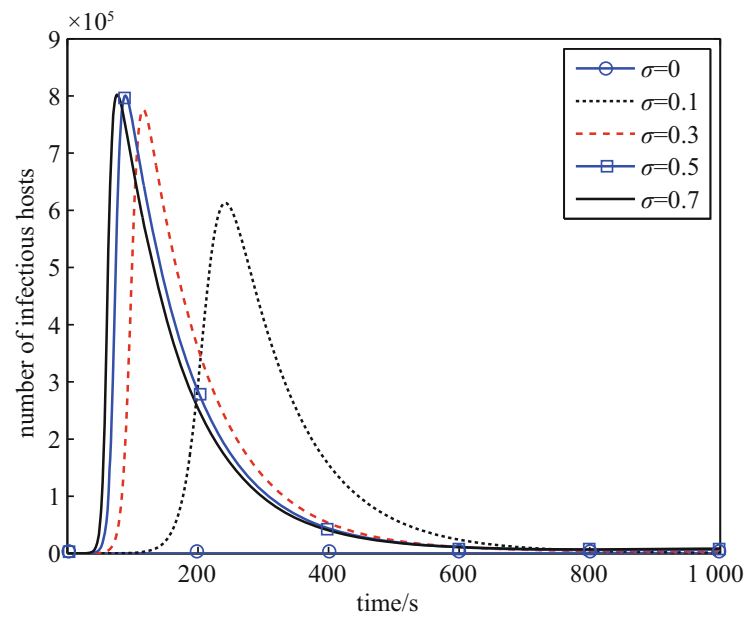

Figure 4 Effect of the partial immunization rate 
immunize their computers in a very short time, which would guarantee them reaching a smaller partial immunization rate $\sigma$.

\section{Conclusion and future work}

This paper presented a novel dynamic SVEIR model for worms. The SVEIR model extends the classical SEIR model by incorporating a saturated incidence rate and a partial immunization rate. More specifically, this study investigated the global dynamic behavior of the SVEIR model by using the Lyapunov function and a geometric approach. The theoretical analysis demonstrated that when the basic reproduction number is smaller than or equal to one, the SVEIR model has an infection-free equilibrium, and is globally asymptotically stable. When the basic reproduction number is larger than one, the SVEIR model has a unique endemic equilibrium and is globally stable. The simulation results are consistent with theoretical analysis. Our proposed SVEIR model is expected to be highly useful to analyze the availability and efficiency of partial immunization, which becomes efficient if the partial immunization rate is very small. This is very helpful to eliminate worms as soon as possible.

In future, we plan to examine how to eliminate worms, quickly prevent their propagation across networks after detecting the worms attack, and expand the proposed SVEIR model to scale-free networks.

\section{References}

[1] ZOU C C, TOWSLEY D, GONG W. On the performance of Internet worm scanning strategies[J]. Performance evaluation, 2006, 63(7): 700-723.

[2] MOORE D, PAXSON V, SAVAGE S, et al. Inside the Slammer worm[J]. IEEE security and privacy, 2003, 1(4): 33-39.

[3] TOUTONJI O A, YOO S M, PARK M. Stability analysis of veisv propagation modeling for network worm attack[J]. Applied mathematical modelling, 2012, 36(6): 2751-2761.
[4] KAUR R, SINGH M. A survey on zero-day polymorphic worm detection techniques[J]. IEEE communications surveys and tutorials, 2014, 16(3): 1520-1549.

[5] MISHRA B K, PANDEY S K. Dynamic model of worms with vertical transmission in computer network[J]. Applied mathematics and computation, 2011, 217(21): 8438- 8446.

[6] PENG S, WU M, WANG G, et al. Propagation model of smartphone worms based on semi-markov process and social relationship graph[J]. Computers \& security, 2014, 44(1): 92-103.

[7] MISHRA B K, Pandey S K. Dynamic model of worm propagation in computer network[J]. Applied mathematical modelling, 2014, 38(7): 2173-2179.

[8] FENG C, YANG J, QIN Z, et al. Modeling and analysis of passive worm propagation in the P2P file-sharing network[J]. Simulation modelling practice and theory, 2015, 51(2): 87-99. [

[9] WANG C, CHAI S. Hopf bifurcation of an SEIRS epidemic model with delays and vertical transmission in the network[J]. Advances in difference equations, 2016, 2016(100): 1-19.

[10] XIAO X, FU P, DOU C, et al. Design and analysis of SEIQR worm propagation model in mobile internet[J]. Communications in nonlinear science and numerical simulation, 2017, 43(1): 341-350.

[11] ZHANG Z, YANG H. Stability and Hopf bifurcation for a delayed SLBRS computer virus model[J]. Scientific world journal, 2014, Artitle ID 373171: 1-12.

[12] KERMACK W, MCKENDRIC K. Contributions of mathematical theory to epidemics $[\mathrm{J}]$. Proceedings of the royal society of London: series A, 1927, 115(772): 700-721.

[13] ARON J L, SCHWARTZ I B. Seasonality and period- doubling bifurcations in an epidemic model[J]. Journal of theoretical biology, 1984, 110(4): 665-679.

[14] Hosseini S, AZGomi M A, RAhMAni A T. Malware propagation modeling considering software diversity and immunization[J]. Journal of computational science, 2016, 12(3): 4967.

[15] LIU W, LIU C, LIU X, et al. Modeling the spread of malware with the influence of heterogeneous immunization[J]. Applied mathematical modelling, 2016, 40(4): 3141-3152.

[16] GAN C, YANG X, LIU W, et al. A propagation model of computer virus with nonlinear vaccination probability $[\mathrm{J}]$. Communications in nonlinear science and numerical simulation, 2014, 19(1): 92-100.

[17] JIA J, LI P. Global analysis of an sveir epidemic model with partial immunity[J]. Mathematica aeterna, 2011, 1(8): 457-561.

[18] OZAAIR M, HUSSAIN T. Analysis of vector-host model with latent stage having partial immunity[J]. Applied mathematical sciences, 2014, 8(32): 1569-1584.

[19] CAI L, LASHARI A A, JUNG I H, et al. Mathematical analysis of a malaria model with partial immunity to re-infection[J]. Abstract and applied analysis, 2013, Article ID 405258: 1-17.

[20] CAPASSO V, SERIO G. A generalization of the kermackmckendrick deterministic epidemic model[J]. Mathematical biosciences, 1978, 42(1): 43-61.

[21] LIU X, YANG L. Stability analysis of an seiqv epidemic model with saturated incidence rate[J]. Nonlinear analysis: real world 
applications, 2012, 13(6): 2671-2679.

[22] LAARABI H, LABRIJI E, RACHIK M, et al. Optimal control of an epidemic model with a saturated incidence rate[J]. Nonlinear analysis: modelling and control, 2012, 17(4): 448-459.

[23] PENG H, GUO Z. Global stability for a viral infection model with saturated incidence rate[J]. Abstract and applied analysis, 2014, Article ID 187897: 1-9.

[24] PORWAL P, BADSHAH V. Modified epidemic model with saturated incidence rate and reduced transmission under treatment[J]. International journal of mathematical archive, 2014, 4(12): 106-111.

[25] VAN DEN DRIESSCHE P, WATMOUGH J. Reproduction numbers and subthreshold endemic equilibria for compartmental models of disease transmission[J]. Mathematical biosciences, 20(1): 29-48.

[26] ROBINSON R C. An introduction to dynamical systems: continuous and discrete[M]. New York: American Mathematical Society Press, 2012.

[27] LASALLE J P. The stability of dynamical systems[M]. Philadelphia, PA: Society for Industrial and Applied Mathematics Press, 1976.

[28] MORRIS J. The Routh and Routh-Hurwitz stability criteria[J]. Aircraft engineering and aerospace technology, 1962, 34(1): 25-27.

[29] MA Z, ZHOU Y, WANG W, et al. Mathematical models and dynamics of infection diseases[M]. Beijing: China Science Press, 2004.

[30] LI M Y, GRAEF G R, WANG L, et al. Global dynamics of a seir model with varying total population size[J]. Mathematical biosciences, 1999, 16(2): 191-213.

\section{About the authors}

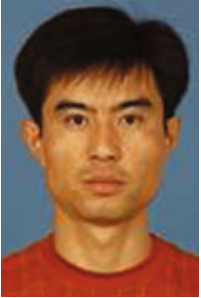

WANG Fangwei was born in 1976. He received his B.S. degree in 2000 from College of Mathematics \& Information Sciences, Hebei Normal University, his M.S. degree in 2003 from College of Computer Science and Software, Hebei University of Technology, his Ph.D. degree in 2009 from College of Computer at Xidian University. Currently he is an associate professor at Hebei Normal University, Shijiazhuang, China. His research interests include: network and information security, sensor networks. (Email: fw wang@hebtu.edu.cn)

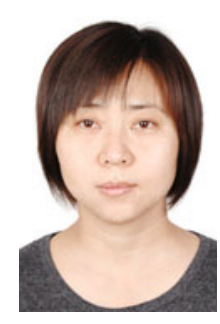

HUANG Wenyan was born in 1978. She received her M.S. degree in 2003 from College of Computer Science and Software, Hebei University of Technology, China. Currently she is a lecture at Hebei Normal University, Shijiazhuang, China. Her research interests include: network and information security. (Email: huangwy@hebtu. edu.cn)

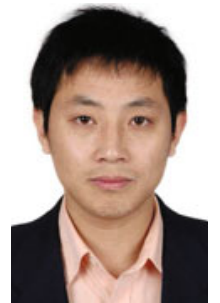

SHEN Yulong was born in 1978. He received the B.E., M.E., and Ph.D. degrees in Computer Science from Xidian University, China, in 2002, 2005, and 2008, respectively. Currently he is professor at Xidian University, Xi'an, China. His research interests include wireless network security and optimization, wireless network information theory, service computing, distributed network storage and security. (Email: ylshen@mail. xidian.edu.cn)

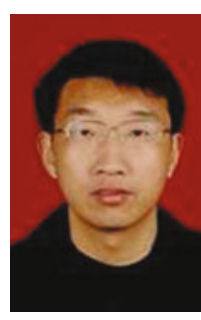

WANG Changguang [corresponding author] was born in 1971. He received his M.S. degree in 1996 from School of Physical Science and Technology, Sichuan University, and his Ph.D. degree in 2009 from College of Computer at Xidian University. Currently he is a professor at Hebei Normal University, Shijiazhuang, China. His research interests include network and information security. (Email: wangcg@hebtu.edu.cn) 